\title{
LETTERS
}

\section{Physical intervention in older adult psychiatry: an audit of physical ailments identified by physiotherapists and the implications for managing aggressive behavior}

Aggression and violence are frequent events in healthcare settings across the globe, with mental health and old age settings often frequenting top spot in incidence lists (Hodgson et al., 2004, Behar et al., 2008). Some may underestimate the challenging nature of older age psychiatric patients, but the reality is that this population can display high levels of aggressive and violent behavior (Stewart et al., 2008). The potentially challenging nature of this population was revealed in a recent study in the U.K., which reported 2753 episodes of aggressive behavior over a three month period (Stewart et al., 2008). Over half of these aggressive displays were in the form of physical aggression, and displays of this type of behavior increase the likelihood of healthcare staff having to employ physical intervention (Stewart et al., 2008). Physical intervention is a form of hands-on physical restraint that involves trained staff employing physical holding techniques to move and restrict the movement of the aggressive patient.

Data on the use of physical intervention in older adult settings are limited, but one recent study reports that physical intervention was employed on 292 occasions on 44 older adults $(n=32$ male; $\mathrm{n}=12$ female) over a three-year period (Stubbs et al., 2008a). Of particular interest were the comparatively lower injury rates to patients $(2 \%)$, which was markedly lower than that reported in some studies in general adult psychiatric settings. This is both surprising and interesting as this is a population that may have many co-morbid physical ailments/limitations that would obviously increase the risks of pain and/or injury when applying physical intervention. This letter reports findings from St Andrews Healthcare in the U.K., a leading provider of psychiatric care for over 500 patients across a range of disciplines. One integral contributory factor to this low injury rate was the involvement of the physiotherapist in the safe application of physical interventions in this population. Stubbs et al. (2008b) state that in populations such as older adults and those with acquired brain injury, a physiotherapist would conduct a neuro-musculoskeletal screening assessment. The purpose of this assessment is to identify any pre-existing physical ailments, which, if present, would increase the likelihood of pain and/or injury in the application of physical restraint (Stubbs et al. 2008b). If on assessment a physical ailment or limitation is identified, the physiotherapist would liaise with the clinical team (especially the physical intervention tutor), and collectively they would consider safe alternatives that reduce the probability of pain and/or injury during the application of physical intervention. Once consensus is established, the wider clinical team are informed and care plans are updated accordingly.

In order to highlight the often complex physical presentation of this population, a retrospective audit was adopted and physiotherapy case notes were reviewed to identify those patients with any physical ailment at any joint on their body. This study was conducted in the Townsend Division of St Andrews Healthcare, which has over 100 beds for older adults with a history of challenging and offending behavior. For the purposes of this letter, the physical ailments/limitations were grouped as follows: bone pathology (e.g. osteoarthritis), neurological (e.g. spasticity) and musculoskeletal (lateral epicondylitis) in origin depending on the primary causative factor. The physiotherapists were asked to use their clinical judgment skills to assign any physical ailments to these categories. In reality it is not always as simple as this, but this coding approach was conducted in an effort to highlight the collective complex physical presentations frequently found in this population. Out of 102 older adult inpatients, 15 had a diagnosed joint pathology, 14 had a neurological impairment at one or more joint, and 18 had a musculoskeletal pathology at one or more joint. Thus, in this sample of 102 older adults, 47 older adult inpatients $(46 \%)$ had a physical ailment which conceivably increases their susceptibility to injury from the application of physical intervention. For those who were diagnosed as having a physical ailment/limitation, adaptive physical intervention techniques were employed to reduce the risk of injuring them. The low injury rates to this older adult sample $(2 \%)$ can be attributed at least in part to the screening assessment by physiotherapists and the latter's role in developing pain-free alternative techniques. This information adds an additional facet to the role of the mental health physiotherapist. 
The data in this letter provide some insights into the complex needs of older adult psychiatric patients. If this sample is representative of a typical older adult population, then other services providing psychiatric care for older adults with similarly high levels of aggressive and challenging behavior may consider adopting a similar model in the safe practice of physical interventions. As this letter reveals, failure to do so may increase the probability of the application of standard physical intervention techniques causing pain and/or injury. Clearly this is something all mental healthcare staff should seek to avoid.

\section{References}

Behar, M. E. et al. (2008). Violence risks in nursing - results from the European NEXT study. Occupational Medicine, $58,107-114$

doi:10.1017/\$1041610209990172

\section{The relationship between elderly suicide rates, population density and room density}

A recent cross-national study reported that elderly suicide rates in both sexes were significantly negatively correlated with household size and the percentage of extended households and positively correlated with the percentage of single-person households (Shah, 2009). Speculative explanations for these findings were based on cultural factors, including (i) a mismatch between the traditional dependence of elderly relatives on their children for emotional and financial support and their children's ability to provide this support (Yip et al., 1998, 2000; Liu et al., 2006); (ii) the unmet traditional expectation of the elderly person being able to live with their children or grandchildren (Yip et al., 2000; Liu et al., 2006); (iii) the effect on the elderly of their children's negative attitudes (Yip et al., 2000); (iv) the migration of children to urban areas or to other countries (Yip and Tan, 1998; Yip et al., 2000); and, (v) the number of available caregivers, household size and family size (Kua et al., 2003). Countries with larger household sizes and a greater number of extended households potentially have a greater number of people available within the household and within close geographical proximity who can contribute positively to these cultural issues, and this may ultimately lead to a reduction in elderly suicide rates - the "emotional proximity" explanation. However, there may also be other explanations for
Hodgson, M. J. et al. (2004). Violence in healthcare facilities: lessons from the Veterans Health Administration. Fournal of the American College of Occupational and Environmental Medicine, 46, 1158-1165.

Stewart, I., Knight, C. and Johnson, C. (2008). Just how challenging can older people be? Part 2: Making the case for specialist services for risky and aggressive behaviour. PSIGE Nezwsletter, 103, 66-75.

Stubbs, B., Yorston, G. and Knight, C. (2008a). Physical intervention to manage aggression in older adults: how often is it employed? International Psychogeriatrics, 20, 855-857.

Stubbs, B., Knight, C. and Yorston, G. (2008b). Physical interventions for managing aggression in mental health: should physiotherapists be involved? International fournal of Therapy and Rehabilitation, 15, 8-12.

Brendon Stubbs, Lead Physiotherapist

Townsend Division, St Andrews Healthcare, Northampton, U.K.

Email: bstubbs@standrew.co.uk

these findings (Shah, 2009). Having more people in a household implies that there are more people to identify suicidal ideation and support the suicidal individual in seeking approprate help. Also, in larger households elderly people are likely to be alone for shorter periods of time, which would reduce the opportunity to implement any suicidal plans. Both these possibilities form part of the "geographical proximity" explanation.

If "emotional proximity" is important then elderly suicide rates should decline not only with larger household size but also with a greater density of people per household room, but not with greater general population density. If "geographical proximity" is important then elderly suicide rates should decline not only with larger household size but also with greater general population density and greater density of people per household room. Therefore, a study with two components was conducted: (i) an examination of the relationship between elderly suicide rates and data on household size from a different source than that used in an earlier study (Shah, 2009); and (ii) the relationship between elderly suicide rates and general population density and density of people per household room.

Data on elderly suicide rates for both sexes in two age-bands - 65-74 years and $75+$ years were ascertained from the World Health Organization website (www.who.int/whosis/mort/table1.cfm). The median (range) of the latest available year for this data from different countries was 2000 (19852003). Data on the average number of people living in a household, density of people per room and general population density were ascertained from 